\title{
Bevacizumab counteracts VEGF-dependent resistance to erlotinib in an EGFR-mutated NSCLC xenograft model
}

\author{
CHINAMI MASUDA, MIEKO YANAGISAWA, KEIGO YOROZU, MITSUE KURASAWA, KOH FURUGAKI, \\ NOBUYUKI ISHIKURA, TOSHIKI IWAI, MASAMICHI SUGIMOTO and KANAME YAMAMOTO
}

\author{
Product Research Department, Kamakura Research Laboratories, \\ Chugai Pharmaceutical Co., Ltd., Kamakura, Kanagawa 247-8530, Japan
}

Received March 22, 2017; Accepted May 24, 2017

DOI: $10.3892 /$ ijo.2017.4036

\begin{abstract}
Erlotinib, an epidermal growth factor receptor (EGFR) tyrosine kinase inhibitor (TKI), shows superior efficacy in patients with non-small cell lung cancer (NSCLC) harboring activating $E G F R$ mutations $\left(E G F R \mathrm{Mut}^{+}\right)$. However, almost all tumors eventually develop resistance to erlotinib. Recently, the Phase II JO25567 study reported significant prolongation of progression-free survival (PFS) by erlotinib plus bevacizumab combination compared with erlotinib in EGFR Mut ${ }^{+}$NSCLC. Herein, we established a preclinical model which became refractory to erlotinib after long-term administration and elucidated the mode of action of this combination. In this model, tumor regrowth occurred after remarkable shrinkage by erlotinib; regrowth was successfully inhibited by erlotinib plus bevacizumab. Tumor vascular endothelial growth factor (VEGF) was greatly reduced by erlotinib in the erlotinib-sensitive phase but significantly increased in the erlotinib-refractory phase despite continued treatment with erlotinib. Although EGFR phosphorylation remained
\end{abstract}

Correspondence to: Kaname Yamamoto, Product Research Department, Kamakura Research Laboratories, Chugai Pharmaceutical Co., Ltd., 200 Kajiwara, Kamakura, Kanagawa 247-8530, Japan

E-mail: yamamotoknm@chugai-pharm.co.jp

Abbreviations: EGFR, epidermal growth factor receptor; VEGF, vascular endothelial cell growth factor; VEGFR, vascular endothelial cell growth factor receptor; TKI, tyrosine kinase inhibitor; ERK, extracellular signal-regulated kinase; STAT3, signal transducer and activator of transcription 3; bFGF, basic fibroblast growth factor; TGF- $\alpha$, transforming growth factor- $\alpha$; PlGF, placental growth factor; G-CSF, granulocyte-colony stimulating factor; IL-6, interleukin-6; CXCL2, chemokine (C-X-C motif) ligand 2; NSCLC, non-small cell lung cancer; $E G F R \mathrm{Mut}^{+}$, activating $E G F R$ mutations; PFS, progression-free survival; MVD, microvessel density; HIFs, hypoxia-inducible transcription factors; TAMs, tumor associated macrophages; MDSCs, myeloid derived suppressor cells

Key words: EGFR mutation, VEGF, bevacizumab, erlotinib suppressed in the erlotinib-refractory phase, phosphorylated extracellular signal-regulated kinase (pERK), phosphorylated AKT, and phosphorylated signal transducer and activator of transcription 3 (pSTAT3) were markedly higher than in the erlotinib-sensitive phase; among these, pERK was suppressed by erlotinib plus bevacizumab. MVD was decreased significantly more with erlotinib plus bevacizumab than with each drug alone. In conclusion, the erlotinib plus bevacizumab combination demonstrated promising efficacy in the B901L xenograft model of EGFR Mut ${ }^{+}$NSCLC. Re-induction of VEGF and subsequent direct or indirect VEGF-dependent tumor growth was suggested as a major mechanism of erlotinib resistance, and erlotinib plus bevacizumab achieved remarkably prolonged antitumor activity in this model.

\section{Introduction}

Erlotinib belongs to the class of molecular targeted drugs designed as epidermal growth factor receptor (EGFR) tyrosine kinase inhibitors (TKIs). It blocks trans-phosphorylation of EGFR and subsequent downstream signaling in pathways such as the mitogen-activated protein kinase (MAPK) pathway, phosphatidylinositol 3-kinase (PI3K)-AKT pathway, and signal transducer and activator of transcription 3 (STAT3) pathway. Erlotinib treatment results in prolonged progression-free survival (PFS) with a median of 10-14 months in patients with non-small cell lung cancer (NSCLC) harboring EGFR exon 19 deletion or L858R mutations (EGFR Mut ${ }^{+}$NSCLC) (1-3). However, despite these clinical benefits, almost all tumors eventually progress due to acquired resistance (4). Recently, several mechanisms of EGFR-TKI resistance have been identified, including EGFR T790M gatekeeper mutation, activation of bypass signals (ERBB2 gene amplification and MET gene amplification), and other mechanisms (transformation to small cell lung cancer, epithelial to mesenchymal transition, and tumor microenvironment-mediated resistance) (5).

Bevacizumab, a humanized monoclonal antibody targeting vascular endothelial growth factor (VEGF), regresses preexisting tumor blood vessels and blocks the formation of new ones $(6,7)$. Furthermore, it normalizes vascular permeability and thereby decreases interstitial fluid pressure so that it improves delivery of co-administered drugs and therapeutic outcomes (8-10). Consequently, bevacizumab prolongs PFS 
and overall survival in advanced NSCLC when administered in combination with standard first-line platinum-based chemotherapies (11).

Since erlotinib and bevacizumab act on two different pathways critical to tumor growth, administering these drugs concomitantly may confer promising clinical benefits to cancer patients with advanced disease $(12,13)$. The Phase II JO25567 study reported that erlotinib plus bevacizumab produced a statistically significant and clinically meaningful prolongation of PFS compared with erlotinib alone in the treatment of EGFR Mut ${ }^{+}$NSCLC (14). Several preclinical studies in various xenograft models have reported on the mechanisms of erlotinib in addition to bevacizumab (15). In those studies, erlotinib was shown to decrease VEGF expression $(16,17)$ and block synthesis of angiogenic proteins such as basic fibroblast growth factor (bFGF) and transforming growth factor- $\alpha$ (TGF- $\alpha$ ) $(12,18)$. Moreover, PTK787, an inhibitor of VEGF receptor (VEGFR) tyrosine kinases, c-Kit, and angiogenesis, was shown to improve delivery of erlotinib into the tumor in a PC-9 xenograft model (19). However, those data show the mechanisms in the erlotinib-sensitive phase, and the mechanism by which the combination of erlotinib and bevacizumab confers prolonged efficacy even into the erlotinib-refractory phase remains to be elucidated.

In the present study, we established a human EGFR Mut NSCLC xenograft model that became refractory in which tumor regrowth was observed by long-term erlotinib administration, and we analyzed the mechanisms of both the erlotinib-sensitive and erlotinib-refractory phases.

\section{Materials and methods}

Test agents. Erlotinib was provided by F. Hoffmann-La Roche Ltd. (Basel, Switzerland) and was dissolved in 6\% Captisol solution (ChemScene, Monmouth Junction, NJ, USA). Bevacizumab was obtained from F. Hoffmann-La Roche Ltd. Human immunoglobulin G (HuIgG) was purchased from MP Biomedicals (Santa Ana, CA, USA). Both bevacizumab and HuIgG were diluted with saline.

Cell lines and culture conditions. B901L (harboring EGFR exon 19 deletion) was purchased from the Institute of Physical and Chemical Research (RIKEN, Saitama, Japan). This cell line was maintained in RPMI-1640 (Sigma-Aldrich, St. Louis, MO, USA) supplemented with $10 \%$ (v/v) fetal bovine serum (Bovogen Biologicals, Melbourne, Australia), $0.45 \%$ D-glucose (Sigma-Aldrich), $10 \mathrm{mM}$ HEPES buffer (Sigma-Aldrich), and $1 \mathrm{mM}$ Na-pyruvate (Thermo Fisher Scientific, Waltham, MA, USA) at $37^{\circ} \mathrm{C}$ under $5 \% \mathrm{CO}_{2}$. NCI-H1975 (harboring T790M mutation) was purchased from ATCC and maintained in RPMI-1640 supplemented with $10 \%$ (v/v) fetal bovine serum at $37^{\circ} \mathrm{C}$ under $5 \% \mathrm{CO}_{2}$.

Animals. Male, 5-week-old BALB/c-nu/nu mice $(\mathrm{CAnN}$. Cg-Foxn $1<$ nu $>/$ CrlCrlj nu/nu) were obtained from Charles River Laboratories Inc. (Kanagawa, Japan). All animals were allowed to acclimatize and recover from shipping-related stress for at least 1 week prior to the study. The health of the mice was monitored by daily observation. The animals were kept under a controlled light-dark cycle (12-12 h), and chlorinated water and irradiated food were provided ad libitum. All animal experiments were reviewed and approved by the Institutional Animal Care and Use Committee at Chugai Pharmaceutical Co., Ltd.

Evaluation of antitumor activity with concurrent treatment. Each mouse was subcutaneously inoculated into the right flank with B901L cells $\left(5 \times 10^{6}\right.$ cells/mouse). After the tumor volume (TV) reached 200-600 $\mathrm{mm}^{3}$, mice were randomly allocated to control and treatment groups, and administration of drugs was started (day 1). Bevacizumab or HuIgG was administered intraperitoneally once a week at a maximum effective dose of $5 \mathrm{mg} / \mathrm{kg}$. Erlotinib or vehicle was orally administered daily at 30 or $60 \mathrm{mg} / \mathrm{kg}$ (maximum effective dose). TV and body weight were measured twice a week. The antitumor activity was evaluated by TV, which was estimated from the equation $\mathrm{TV}=a b^{2} / 2$, where $\mathrm{a}$ and $\mathrm{b}$ are tumor length and width, respectively. Tumor regrowth was defined as an increase in TV at the final observation date compared to the day of minimum TV. Complete tumor regression was defined as a tumor volume below the limit of detection of $<15 \mathrm{~mm}^{3}$.

Evaluation of antitumor activity with bevacizumab add-on treatment after becoming erlotinib-refractory. B901L cells $\left(5 \times 10^{6}\right.$ cells/mouse) were inoculated subcutaneously into the right flank of the mice. After TV reached 200-600 $\mathrm{mm}^{3}$, mice were administered erlotinib orally daily from day 1 until day 63 . The individual mice which tumor regrowth was observed was re-randomized on day 64 and allocated to erlotinib, bevacizumab, or combination of erlotinib plus bevacizumab groups. Erlotinib or vehicle was administered orally daily from day 64 to day 78. Bevacizumab or HuIgG was administered intraperitoneally once a week from day 64 to day 78 . To evaluate the antitumor effect, TV and body weight were measured twice a week. The antitumor activity was evaluated by TV ratio, which was calculated by the equation $a / b$, where $a$ is the TV each day and $b$ is the TV on day 64 because the TV of the largest tumor in the re-randomized group on day 64 was more than twice that of the smallest tumor. The relative TV mean of day 22 became minimum. Complete tumor regression was defined as tumor volume under detection limit $<15 \mathrm{~mm}^{3}$.

Tumor tissue homogenates. Tumor tissues were collected $3 \mathrm{~h}$ after erlotinib administration on indicated days and were immediately frozen in liquid nitrogen and stored at $-80^{\circ} \mathrm{C}$ until use. The tumor tissues were homogenized with Cell Lysis Buffer (Cell Signaling Technology, Danvers, MA, USA) containing NaF (Sigma-Aldrich), Complete Protease Inhibitor Cocktail Tablets (Roche Diagnostics, Tokyo, Japan), and PhosSTOP (Roche Diagnostics). Following centrifugation, the resultant supernatant was used for the assays. Total protein concentration of the supernatant was quantified with a Direct Detect spectrometer (Merck Millipore, Darmstadt, Germany).

Western blotting. Tumor tissue homogenate supernatants (20 $\mu \mathrm{g}$ protein/lane) were electrophoresed on SDS-PAGE and transferred to a polyvinylidene difluoride membrane using an iBlot Gel Transfer Device (Thermo Fisher Scientific). The membrane was blocked in Blocking One (Nacalai Tesque, Kyoto, Japan) and was primarily treated with antibodies to EGFR, STAT3, AKT, ERK, pEGFR, pAKT, and 


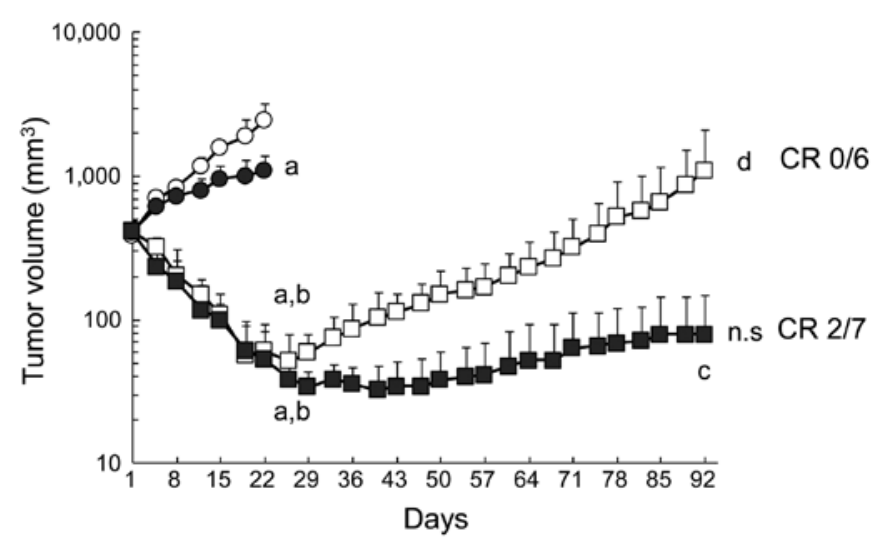

Figure 1. Prolonged antitumor efficacy of erlotinib plus bevacizumab B901L xenograft-bearing mice were continuously treated with control (HuIgG plus vehicle, open circles), $5 \mathrm{mg} / \mathrm{kg}$ of bevacizumab (closed circles), $60 \mathrm{mg} / \mathrm{kg}$ of erlotinib (open squares), or erlotinib plus bevacizumab (combination, closed squares) $(\mathrm{n}=6$ or 7$)$. Each point represents the mean $+\mathrm{SD}$ a, $\mathrm{P}<0.05$ versus control on day $22 ; \mathrm{b}, \mathrm{P}<0.05$ versus bevacizumab on day 22; c, $\mathrm{P}<0.05$ versus erlotinib on day 92 (by Wilcoxon test). d, $\mathrm{P}<0.05$ versus erlotinib on day 26 ; n.s., $P>0.05$ versus combination on day 40 when the mean $\mathrm{TV}$ of each group reached its respective minimum (by Wilcoxon test). CR, complete tumor regression.

pERK (Cell Signaling Technology), antibody to pSTAT3 (Abcam, Cambridge, MA, USA), and antibody to $\beta$-actin (Sigma-Aldrich). These proteins were detected by horseradish peroxidase-conjugated secondary antibodies (Cell Signaling Technology) and ECL Prime Western Blotting Detection Reagents (GE Healthcare Life Sciences, Little Chalfont, UK). ImageQuant 400 (GE Healthcare Life Sciences) was used for detection, and ImageQuant TL Software was used to digitize the strength of bands.

ELISA analysis. The concentrations of human VEGF, bFGF, placental growth factor (PIGF), TGF- $\alpha$, granulocyte-colony stimulating factor (G-CSF), and interleukin-6 (IL-6) in homogenates of tumor tissues were evaluated by using Quantikine ELISA (R\&D Systems, Minneapolis, MN, USA) following the manufacturer's instructions. The human chemokine (C-X-C motif) ligand 2 (CXCL2) concentrations in tumor tissues were evaluated by ELISA kit (Abnova, Taipei, Taiwan) following the manufacturer's instructions. A Benchmark Plus Microplate Reader (Bio-Rad, Tokyo, Japan) was used for detection.

Ki-67 staining. Proliferating cells were assessed with immunohistochemical staining of Ki-67 (mouse anti-human Ki-67 monoclonal antibody; Agilent Technologies, Glostrup, Denmark). B901L tumors were collected on day 6 after initiation of the treatment. The tissues were fixed with $10 \%$ neutral buffered formalin, and embedded in paraffin. Ki-67 staining was performed and the number of $\mathrm{Ki}-67^{+}$tumor cells in 1000 tumor cells was counted by Sapporo General Pathology Laboratory Co., Ltd. (Sapporo, Japan).

Quantification of microvessel density in tumor tissues. Microvessel density (MVD) in tumor tissues was evaluated by immunohistochemical staining of CD31 (rat anti-mouse CD31 monoclonal antibody; BD Biosciences, San Jose, CA, USA).
Tumor samples from freshly frozen tissues were collected on indicated days. MVD (\%) was calculated from the ratio of the CD31-positive staining area to the total observation area in the viable region. Three to six fields per section were randomly analyzed, excluding necrotic areas. Positive staining areas were calculated by using imaging analysis software (WinROOF; Mitani Corporation, Fukui, Japan).

Melting curve analysis. Melting curve analysis was performed as previously described (20). In brief, to analyze T790M mutation status, exon 20 of EGFR was first amplified by PCR from DNA by using the appropriate primers and the LightCycler 480 Genotyping Master (Roche Diagnostics, Mannheim, Germany), and then hybridized by using sensor and anchor probes. Human genomic DNA (Promega, Madison, WI, USA) was used as a wild-type EGFR control.

Statistical analysis. Differences were considered statistically significant for values of $\mathrm{P}<0.05$ by the t-test or Wilcoxon's rank sum test using JMP version 11 software (SAS Institute, Cary, NC, USA). Data are represented as means and SD.

\section{Results}

Combined administration of erlotinib plus bevacizumab resulted in prolonged antitumor efficacy in B901L xenograft model. First, we evaluated the dose response of erlotinib in the B901L xenograft model. Although significant tumor growth inhibition was observed in mice treated with $30 \mathrm{mg} / \mathrm{kg}$, drug efficacy was moderate and did not result in significant tumor shrinkage (data not shown). In mice treated with $60 \mathrm{mg} / \mathrm{kg}$, on the other hand, remarkable tumor shrinkage was observed in the initial phase. However, tumor regrowth was observed despite continued erlotinib administration (Fig. 1). It is known that almost all patients eventually acquire resistance to EGFR-TKIs within a few years (4), and with this B901L xenograft model we successfully established a model that could replicate this acquired resistance to erlotinib by prolonged treatment.

Next, we investigated the effects of combined administration of bevacizumab in this model. In distinction to erlotinib, no significant tumor regrowth was observed in the erlotinib plus bevacizumab group at the final observation date. Complete tumor regression, which was defined as a tumor volume below the detection limit, was observed in 2/7 mice treated with erlotinib plus bevacizumab, whereas complete tumor regression was not observed in any mice treated with erlotinib alone.

Erlotinib and bevacizumab exhibit stronger inhibition of MVD and tumor cell proliferation in the erlotinib-sensitive phase. In the erlotinib-sensitive phase, remarkable tumor regression was observed with both the erlotinib and the erlotinib plus bevacizumab treatments. Even though there was no difference in tumor volume in the erlotinib-sensitive phase, afterwards a superior antitumor effect was observed and complete tumor regression was observed in some mice in the erlotinib plus bevacizumab group in contrast to the tumor regrowth seen in the erlotinib group (Fig. 1). Therefore, we supposed that cellular changes had already occurred in the tumor even in the earlier phase. 


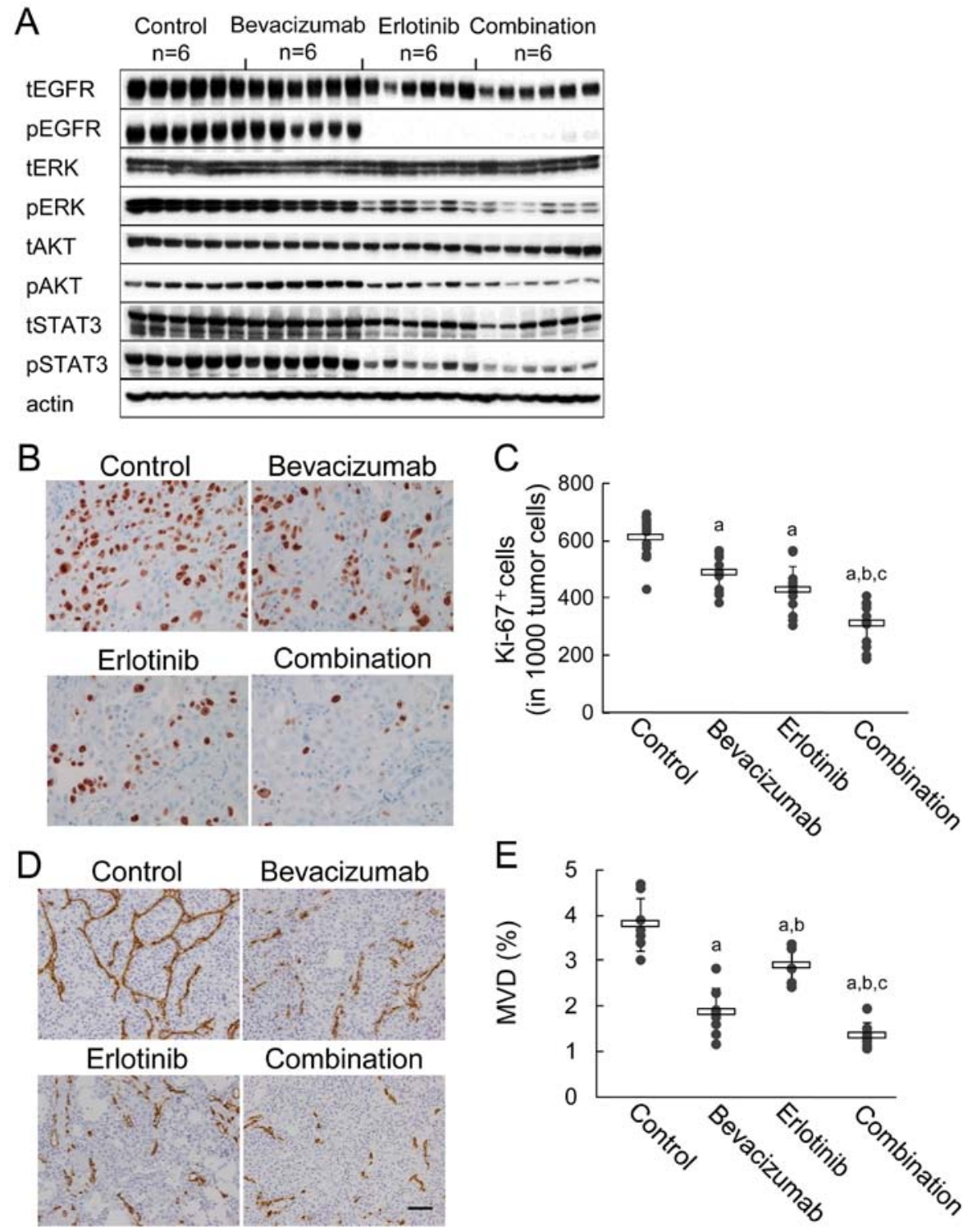

Figure 2. Effect of erlotinib and bevacizumab on signal transduction and histological analyses. (A) Immunoblots of tumor lysates $3 \mathrm{~h}$ after treatment with control, $5 \mathrm{mg} / \mathrm{kg}$ of bevacizumab, $60 \mathrm{mg} / \mathrm{kg}$ of erlotinib, or erlotinib plus bevacizumab combination. Tumor samples ( $\mathrm{n}=6$ ) were collected on day 5 . Total EGFR (tEGFR), phosphorylated EGFR (pEGFR), total ERK (tERK), phosphorylated ERK (pERK), total AKT (tAKT), phosphorylated AKT (pAKT), total STAT3 (tSTAT3), phosphorylated STAT3 (pSTAT3), and actin were detected by a manual western blotting method. (B) Representative images of tumor samples $(n=12)$ sectioned on day 6 and stained with Ki-67. (C) Number of Ki-67+ tumor cells per 1000 tumor cells. (D) MVD in tumor tissues was determined by CD31-immunostaining. Representative immunohistochemical images of tumor samples collected on day 4 ( $\mathrm{n}=5$ or 8 ). Scale bar is $100 \mu \mathrm{m}$. (E) MVD in the control, bevacizumab, erlotinib, and erlotinib plus bevacizumab groups. Bars represent mean $\pm \mathrm{SD}$. a, $\mathrm{P}<0.05$ versus control; $\mathrm{b}, \mathrm{P}<0.05$ versus bevacizumab; c, $\mathrm{P}<0.05$ versus erlotinib (by Wilcoxon test).

First we examined the effect on signal transduction by using tumor tissues obtained on day 5. EGFR and downstream ERK, AKT, and STAT3 phosphorylation were inhibited in the erlotinib and erlotinib plus bevacizumab groups. On the other hand, inhibition of signal transduction was not detected in the bevacizumab group (Fig. 2A).

Next, we determined whether there was any difference in tumor cell proliferation in tumor tissues sampled on day 6 . Compared with control, the number of $\mathrm{Ki}-67^{+}$tumor cells in the area was decreased by each single agent. With erlotinib plus bevacizumab, it was further decreased compared with each drug alone (Fig. 2B). The number of $\mathrm{Ki}-67^{+}$tumor cells per 1000 tumor cells (mean \pm SD) was also decreased significantly in the erlotinib plus bevacizumab group $(308 \pm 74)$ compared to that in the control $(610 \pm 74)$, bevacizumab $(488 \pm 63)$, and erlotinib (426 \pm 84$)$ groups $(\mathrm{P}<0.05)$ (Fig. 2C).

Then, MVD in tumor tissues was evaluated using specimens obtained on day 4. MVD (\%; mean \pm SD) of the control, bevacizumab, erlotinib, and erlotinib plus bevacizumab groups was, respectively, $3.79 \pm 0.58,1.85 \pm 0.53,2.88 \pm 0.43$, and $1.35 \pm 0.28$, indicating that erlotinib plus bevacizumab suppressed MVD compared with each drug alone $(\mathrm{P}<0.05)$ (Fig. 2D and E). Among the cytokines with pro-angiogenic activities that we tested, IL-6, G-CSF, and CXCL2 were inhibited by erlotinib, whereas bFGF and TGF- $\alpha$ were not (Table I).

Re-induction of tumor VEGF was involved in the erlotinib resistance mechanism and was inhibited by bevacizumab. 
A

A

Day 5

Day 75

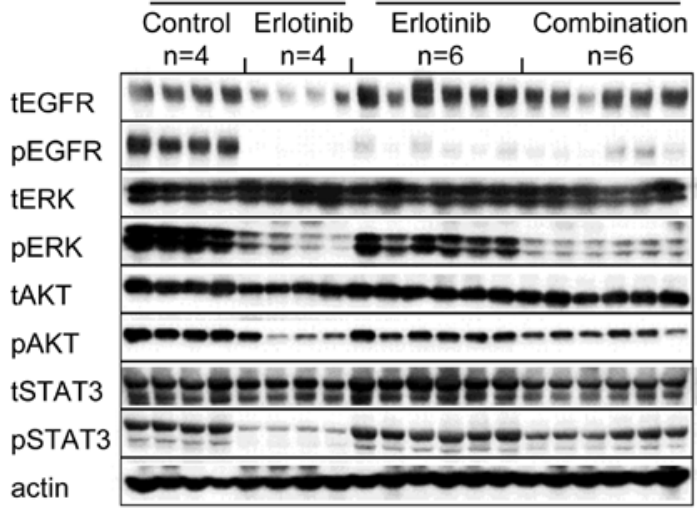

B
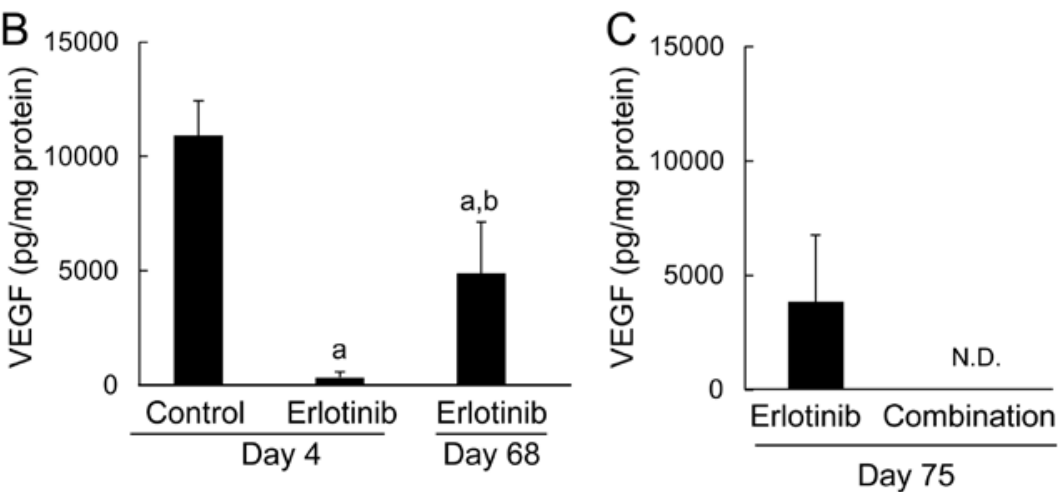

D

Day 15

E

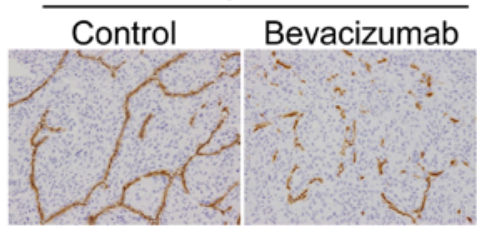

Day 78
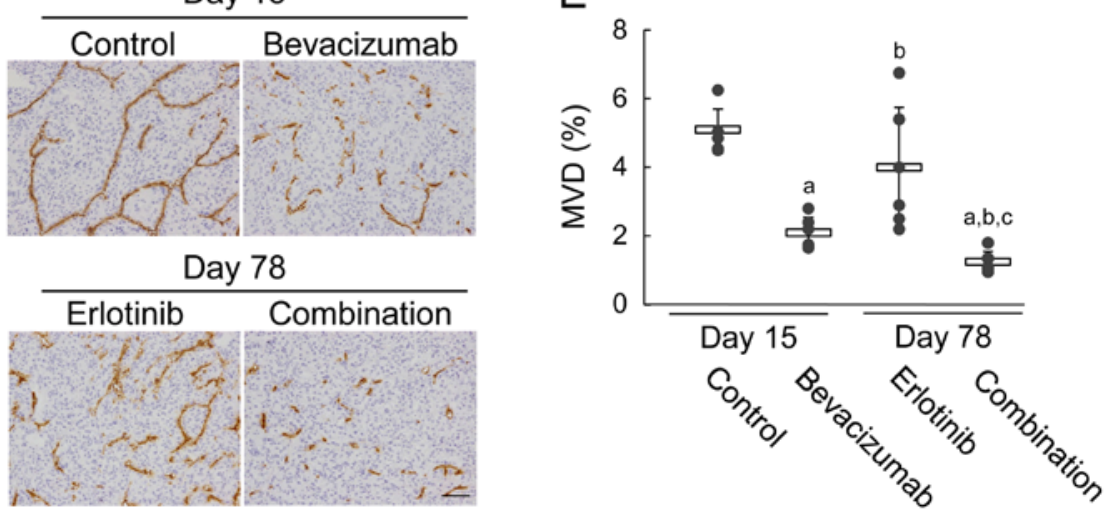

Figure 3. Re-induction of tumor VEGF protein in the erlotinib-refractory phase, and erlotinib plus bevacizumab-induced suppression of MVD and signaling pathways. (A) Immunoblots of tumor lysates $3 \mathrm{~h}$ after treatment with control, $5 \mathrm{mg} / \mathrm{kg}$ of bevacizumab, $60 \mathrm{mg} / \mathrm{kg}$ of erlotinib, or erlotinib plus bevacizumab combination. Tumor samples ( $\mathrm{n}=4$ or 6 ) were collected on day 5 (control or erlotinib) or day 75 (erlotinib or combination). Total EGFR (tEGFR), phosphorylated EGFR (pEGFR), total ERK (tERK), phosphorylated ERK (pERK), total AKT (tAKT), phosphorylated AKT (pAKT), total STAT3 (tSTAT3), phosphorylated STAT3 (pSTAT3), and actin were detected by a manual western blotting method. (B) Levels of VEGF protein expression in tumors after $3 \mathrm{~h}$ of treatment with control or $60 \mathrm{mg} / \mathrm{kg}$ of erlotinib. Tumor samples were collected on day 4 (control or erlotinib) or day 68 (erlotinib) (n=5 or 6 ). Bars represent the mean $+\mathrm{SD}$. a, $\mathrm{P}<0.05$ versus control; $\mathrm{b}, \mathrm{P}<0.05$ versus day 4 erlotinib (by Wilcoxon test). (C) Tumor samples were collected on day 75 (erlotinib or combination) ( $=4$ ). (D) Representative immunohistochemical images of CD31-immunostaining to determine MVD in tumor tissues from mice treated with control, 5 mg/kg of bevacizumab, $60 \mathrm{mg} / \mathrm{kg}$ of erlotinib, or combination. Tumor samples $(\mathrm{n}=6)$ were collected on day 15 (control or bevacizumab) or day 78 (erlotinib or combination). Scale bar is $100 \mu \mathrm{m}$. (E) MVD in the control, bevacizumab, erlotinib, and erlotinib plus bevacizumab groups. Bars represent mean $\pm \mathrm{SD}$. a, $<<0.05$ versus control; $\mathrm{b}, \mathrm{P}<0.05$ versus bevacizumab; $\mathrm{c}, \mathrm{P}<0.05$ versus erlotinib (by Wilcoxon test).

As shown in Fig. 1, tumor became refractory and regrowth was observed in the erlotinib group. Therefore, we compared the status of signal transduction using specimens collected in the erlotinib-sensitive and erlotinib-refractory phases. In the erlotinib-sensitive phase (day 5), pEGFR, pERK, pAKT, and pSTAT3 were strongly decreased. In contrast, although pEGFR was still suppressed in the erlotinib group in the erlotinib-refractory phase (day 75), pERK, pAKT, and pSTAT3 were increased compared with levels on day 5 (Fig. 3A). This finding implied that there was a resistance mechanism other than that occurring in EGFR itself, although the T790M mutation was not detected in this model (Fig. 4).

Next, we quantified human VEGF concentrations by using tumor specimens obtained in the erlotinib-sensitive and erlotinib-refractory phases. In the erlotinib-sensitive phase (day 4), human VEGF concentrations were reduced significantly compared to concentrations in the control group. Interestingly, human VEGF levels in the erlotinib-refractory 


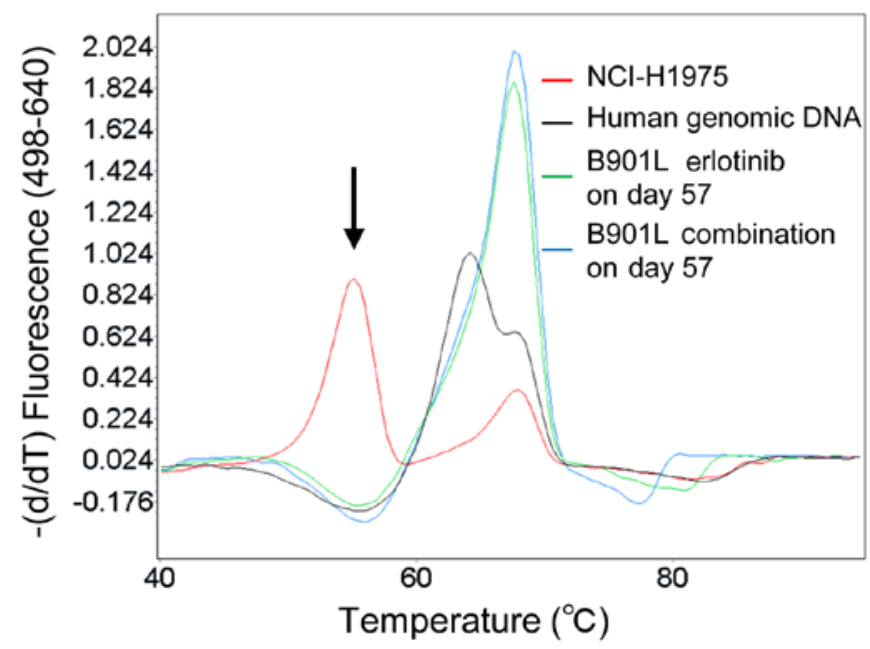

Figure 4. Analysis of T790M mutation in tumors treated with erlotinib or erlotinib plus bevacizumab in the erlotinib-refractory phase. Xenograftbearing mice were treated with erlotinib (green) or combination (blue) for 57 days. The content ratio of the T790M mutation in the B901L model was analyzed by the melting curve method. Human genomic DNA extracted from whole blood from multiple anonymous normal donors was used as a wildtype EGFR control (grey). NCI-H1975 was used as a positive control (red). The peak of the T790M mutation is shown (arrow).

phase (day 68) were increased $(\mathrm{P}<0.05)$ compared with levels in the erlotinib-sensitive phase (day 4) (Fig. 3B). In contrast, levels of free human VEGF in the erlotinib plus bevacizumab group on day 75 were below the detection limit (Fig. 3C). pERK was decreased strongly and pAKT and pSTAT3 tended to be suppressed in the erlotinib plus bevacizumab group compared to in the erlotinib group (day 75) (Fig. 3A).

Then we evaluated MVD in tumor tissues. Significant suppression of MVD was not observed by erlotinib in the erlotinib-refractory phase on day 78 compared with control on day 15 (Fig. 3D and E) although MVD was suppressed by erlotinib on day 4 compared with control in its sensitive phase $(\mathrm{p}<0.05)$ (Fig. 2E). Mean MVD (\%) in the bevacizumab group $(2.06 \pm 0.47)$ was inhibited compared with mean MVD in the control group on day 15 (5.04 \pm 0.65$)$. Furthermore, MVD was significantly more suppressed by erlotinib plus bevacizumab on day $78(1.20 \pm 0.32)$ compared with bevacizumab on day 15 (Fig. 3D and E). In contrast to VEGF levels, the levels of bFGF, TGF- $\alpha$, G-CSF, and CXCL2 in the erlotinib-refractory phase were not increased compared with their levels in the erlotinibsensitive phase (Table I). Although a significant increase in IL-6 was observed in the erlotinib-refractory phase compared to levels in the erlotinib-sensitive phase, the levels of IL-6 were much lower than in the control group (Table I). Taken together, these results suggest that VEGF is involved, at least in part, in erlotinib resistance mechanisms. Furthermore, the antiangiogenic effect and the inhibition of signal transduction were suggested to be mechanisms underlying the antitumor activity of the combination of erlotinib plus bevacizumab.

Inhibition of VEGF after establishment of refractoriness to erlotinib showed significant but limited antitumor efficacy. Since the data described above suggested the possibility that tumor VEGF, the production of which reappeared even under the presence of erlotinib, was the key molecule behind tumor
Table I. Levels of angiogenic factors in different phases of erlotinib treatment.

\begin{tabular}{lcccc}
\hline & \multicolumn{2}{c}{$\begin{array}{c}\text { Erlotinib-sensitive } \\
\text { phase }\end{array}$} & & $\begin{array}{c}\text { Erlotinib-refractory } \\
\text { phase }\end{array}$ \\
\cline { 2 - 3 } $\begin{array}{l}\text { Angiogenic } \\
\text { factor }\end{array}$ & Control & Erlotinib & & Erlotinib \\
\cline { 2 - 3 } bFGF & $194 \pm 58.6$ & $189 \pm 104$ & & $201 \pm 78.6$ \\
PlGF & N.D. & N.D. & & N.D. \\
TGF- $\alpha$ & $34.1 \pm 7.59$ & $31.1 \pm 8.32$ & & N.D. \\
G-CSF & $269 \pm 154$ & $62.4 \pm 59.3^{\text {a }}$ & & $99.8 \pm 76.4$ \\
IL-6 & $924 \pm 1022$ & $22.6 \pm 8.94^{\text {a }}$ & & $80.3 \pm 51.1^{\text {a,b }}$ \\
CXCL2 & $223 \pm 43.8$ & $19.9 \pm 4.54^{\text {a }}$ & & $29.7 \pm 4.29^{\text {a }}$ \\
\hline
\end{tabular}

Levels of bFGF, PlGF, TGF- $\alpha$, G-CSF, IL-6, and CXCL2 protein expression (mean $\pm \mathrm{SD} ; \mathrm{pg} / \mathrm{mg}$ protein) in tumors after $3 \mathrm{~h}$ of treatment with control or $60 \mathrm{mg} / \mathrm{kg}$ of erlotinib. Tumor samples were collected in the erlotinib-sensitive phase on day 4 or day 5 (control or erlotinib) or in the erlotinib-refractory phase on day 68 or day 72 (erlotinib) ( $\mathrm{n}=5$ or 6$)$. ${ }^{\mathrm{a}} \mathrm{P}<0.05$ versus control in the erlotinib-sensitive phase; ${ }^{\mathrm{b}} \mathrm{P}<0.05$ versus erlotinib in the erlotinib-sensitive phase (by Wilcoxon test). N.D., no data.

regrowth, we examined the effect of VEGF inhibition by bevacizumab after the tumors became refractory to erlotinib. After tumor regrowth was observed, mice were re-randomized on day 64 and allocated to receive erlotinib, bevacizumab, or the combination of erlotinib plus bevacizumab. Tumor growth was inhibited by erlotinib plus bevacizumab compared with erlotinib, although complete tumor regression was not observed (Fig. 5A). Furthermore, pERK was suppressed and pAKT and pSTAT3 tended to be suppressed by erlotinib plus bevacizumab compared with erlotinib (Fig. 5B). MVD was also inhibited by erlotinib plus bevacizumab compared with erlotinib (Fig. 5C and D).

\section{Discussion}

In the B901L xenograft model, tumor regrowth was observed following initial strong tumor regression by erlotinib monotherapy (Fig. 1). In the erlotinib-sensitive phase, pEGFR and its downstream pERK, pAKT, and pSTAT3 were suppressed by erlotinib (Fig. 2A), and the expression of tumor VEGF was decreased significantly compared with control (Fig. 3B) in agreement with previously reported downregulation of VEGF by EGFR-TKIs $(16,17,21)$. On the other hand, pERK, pAKT, and pSTAT3 were increased in the erlotinib-refractory phase compared with that in the erlotinib-sensitive phase, although pEGFR was still suppressed (Fig. 3A). The T790M mutation, which is one of the major erlotinib resistance mechanisms occurring in the EGFR tyrosine kinase domain and causing a relative decrease in binding with EGFR-TKIs, was not detected after progression in this model (Fig. 4). Accordingly, it is suggested that bypass pathways other than EGFR activate signaling pathways leading to tumor regrowth. To date, MET amplification, ERBB2 amplification, and overexpression of hepatocyte growth factor (HGF), a ligand for MET, 
A
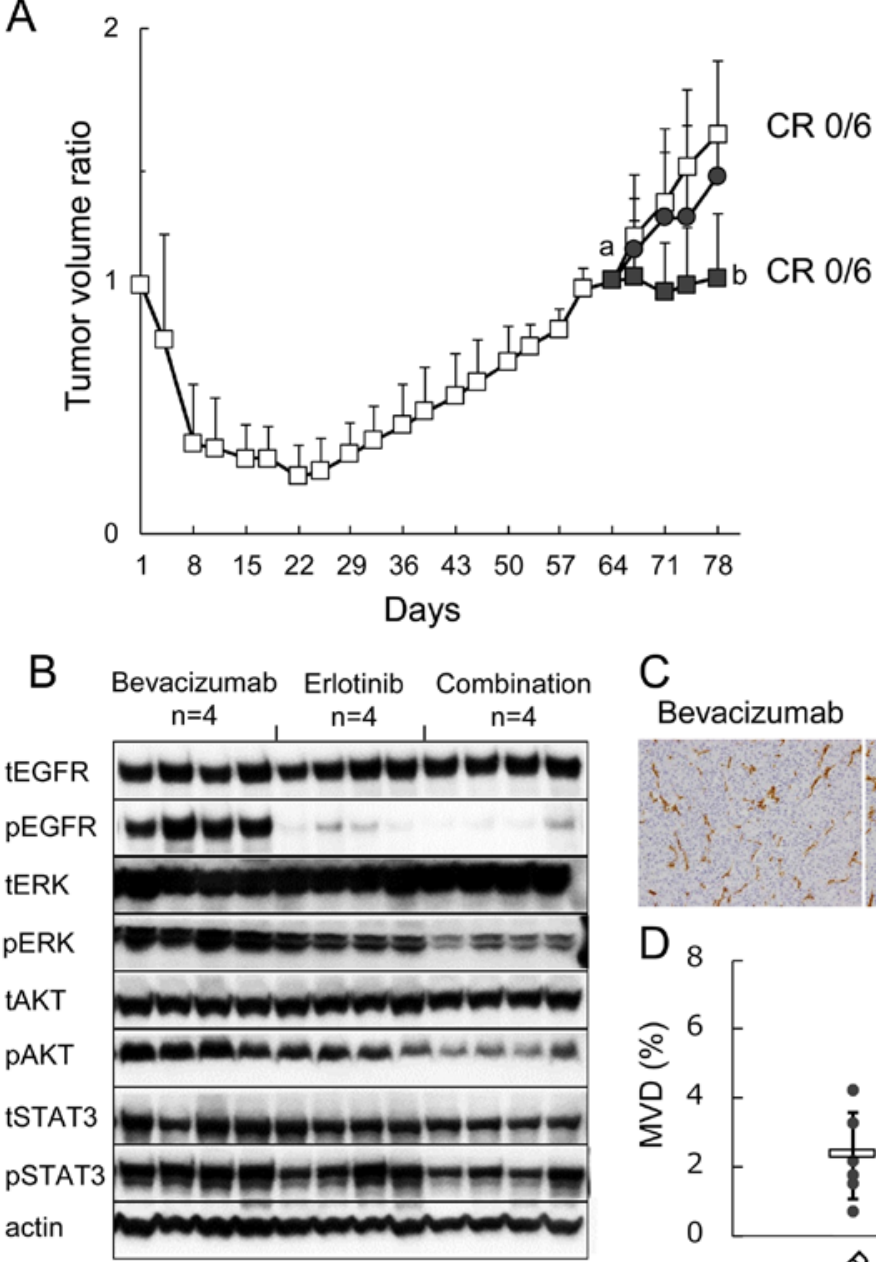

C
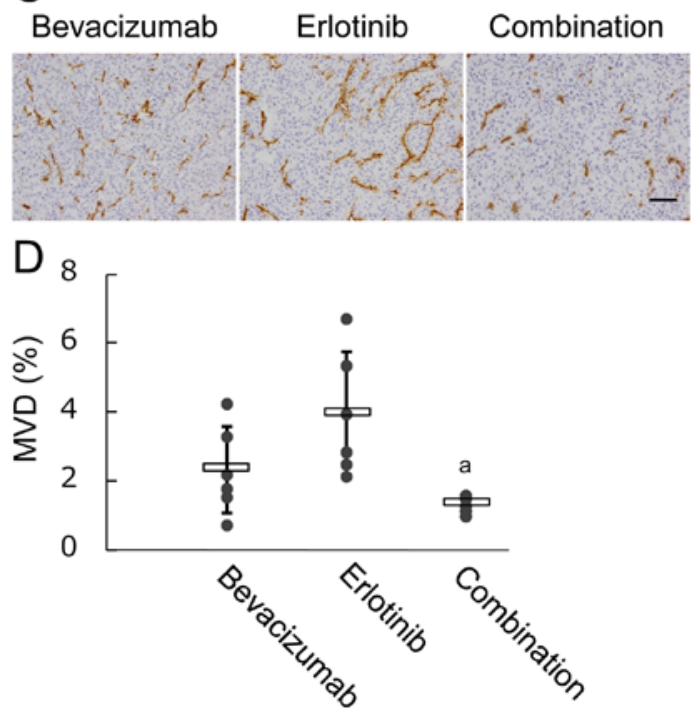

Figure 5. Effect of bevacizumab treatment after establishing erlotinib refractoriness on signaling pathways, MVD, and subsequent tumor growth. (A) B901L xenograft-bearing mice were continuously treated with $60 \mathrm{mg} / \mathrm{kg}$ of erlotinib (open squares). Tumor re-growth was observed during further erlotinib treatment, and mice were re-randomized into 3 groups on day $64(\mathrm{n}=6)$. Mice were treated with $5 \mathrm{mg} / \mathrm{kg}$ of bevacizumab (closed circles), $60 \mathrm{mg} / \mathrm{kg}$ of erlotinib (open squares), or combination (closed squares) $(\mathrm{n}=6)$. Each bar represents the mean $+\mathrm{SD}$. a, $\mathrm{P}<0.05$ versus TV ratio of erlotinib-treated group on day $22 ; \mathrm{b}, \mathrm{P}<0.05$ versus TV ratio of erlotinib-treated group on day 78 (Wilcoxon test). (B) Immunoblots of tumor lysates $3 \mathrm{~h}$ after treatment with bevacizumab, erlotinib, or combination. Tumor samples $(\mathrm{n}=4)$ were collected on day 78. Total EGFR (tEGFR), phosphorylated EGFR (pEGFR), total ERK (tERK), phosphorylated ERK (pERK), total AKT (tAKT), phosphorylated AKT (pAKT), total STAT3 (tSTAT3), phosphorylated STAT3 (pSTAT3), and actin were detected by a manual western blotting method. (C) Representative immunohistochemical images of CD31-immunostaining to determine MVD in tumor tissues collected on day 78 $(\mathrm{n}=6)$. Scale bar is $100 \mu \mathrm{m}$. (D) MVD in the bevacizumab, erlotinib, and erlotinib plus bevacizumab groups. Bars represent mean $\pm \mathrm{SD}$. (A) $\mathrm{P}<0.05$ versus erlotinib (by Wilcoxon test).

K-RAS mutation and PTEN loss have been proposed as erlotinib resistance mechanisms $(5,22)$. However, no change in MET or ERBB2 gene expression nor in HGF protein level were observed and K-RAS mutation was not detected in the tumors in this model (data not shown). Regarding PTEN loss, the amount of pAKT was significantly reduced by erlotinib in earlier sensitive phase and it was increased in refractory phase suggesting activation of PI3K-AKT pathway. Although PTEN loss was considered to be one of its mechanisms, pAKT was decreased by addition of bevacizumab even after acquisition of erlotinib resistance so that we considered it unlikely that observed increase in pAKT was caused by gene mutation or deletion such as PTEN loss. On the other hand, tumor VEGF was markedly restored in the erlotinibrefractory phase, although most of the VEGF production was suppressed by EGFR during the erlotinib-sensitive phase (Fig. 3B).

VEGF expression is driven by many factors that are characteristic of tumors, including oncogene expression, e.g. ras, src, $E R B B 2$, and $E G F R$, and hypoxia (18). Under hypoxia, VEGF is principally regulated by hypoxia-inducible transcription factors (HIFs) (23). Although the mechanism leading to VEGF re-induction in the erlotinib-refractory phase has not yet been fully investigated in this model, in the erlotinib-sensitive phase, MVD was suppressed significantly by erlotinib (Fig. 2D and E), at least in part via downregulation of EGFR-mediated tumor VEGF production, so there is a possibility that hypoxia in the tumor induced by erlotinib and subsequent HIF1 $\alpha$ activation may lead to EGFR-independent VEGF expression. On the other hand, besides EGFR, the HER 
family has also been indicated as playing a role with regard to VEGF regulation (23). For example, monoclonal antibodies targeting HER2 attenuated VEGF expression (24), while VEGF production was enhanced in tumor cells exposed to the HER3/HER4 ligand heregulin (25). In the erlotinib-treated group, pHER2 was activated in the erlotinib-refractory phase but not in the erlotinib-sensitive phase in almost all mice (data not shown). Therefore, HER activation and signaling might also induce VEGF expression.

MVD and signaling pathways were also augmented in the erlotinib-refractory phase suggesting that VEGFmediated angiogenesis and bypass signal activation leads to erlotinib resistance. Indeed, as shown in Fig. 5, inhibition by bevacizumab of the re-induced VEGF production after the erlotinib-refractory phase decreased MVD and signal transduction leading to inhibition of tumor growth. Taken together, these results indicate that VEGF may be a key molecule in erlotinib resistance. Association of VEGF production and erlotinib resistance has also been reported in studies using EGFR wild-type tumor cells. For example, constitutive VEGF up-regulation in anti-EGFR-resistant variants of A431 squamous cell carcinoma was reported (26), and erlotinib resistance was associated with a rise in both tumor cell VEGF and host stromal VEGF in A549 epithelial carcinoma (27).

Of note, although complete tumor regression was observed when erlotinib plus bevacizumab was administered from day 1 , complete regression was not observed by bevacizumab add-on after the erlotinib-refractory phase. Furthermore, although there was no significant difference between TV in the erlotinib group and TV in the erlotinib plus bevacizumab group in the erlotinib-sensitive phase, the number of $\mathrm{Ki}-67^{+}$cells and MVD were decreased in the erlotinib plus bevacizumab group as compared with in the erlotinib group (Fig. 2C and E). Schicher et al showed that bevacizumab decreased the number of $\mathrm{Ki}-67^{+}$cells in tumors (28). These results suggest that the reduction in the number of proliferating cells and MVD in the tumors, which was caused by co-administration of bevacizumab during the erlotinib-sensitive phase, led to the inhibition of regrowth. Furthermore, MVD and pERK were inhibited throughout the study period in the erlotinib plus bevacizumab group (Figs. 2A and E; 3A and E). Taken together, these results indicate that co-administration of bevacizumab both in the erlotinib-sensitive phase and in the erlotinib-refractory phase achieves maximum efficacy. Although pAKT also tended to be suppressed by co-administration of bevacizumab in erlotinibrefractory phase, the level of pAKT was slightly higher than that in erlotinib-sensitive phase. It was suggested that unidentified upstream factors besides VEGF contributed to this modest activation of pAKT.

MVD was suppressed significantly more strongly in the erlotinib plus bevacizumab group compared with in the group administered bevacizumab alone (Figs. 2E and 3E). Since erlotinib has been reported to block production of the angiogenic factors bFGF and TGF- $\alpha$ in addition to $\operatorname{VEGF}(18,29)$, we investigated if other angiogenic factors besides VEGF were suppressed with erlotinib. In this model, the levels of bFGF and TGF- $\alpha$ were not affected by erlotinib and did not increase in the erlotinib-refractory phase. On the other hand, human G-CSF and human CXCL2, which were reported to have proangiogenic activities (30-33), were inhibited by erlotinib in erlotinib-sensitive phase and inhibition of human CXCL2 was statistically significant until the end of study period (Table I). These results suggested that EGFR signal is the inducer of pro-angiogenic factors such as CXCL2 production by tumor throughout the study period in contrast to VEGF, which is induced by other mechanism in erlotinib-refractory phase.

Inhibition of VEGF by bevacizumab and inhibition of other angiogenic factors besides VEGF by erlotinib led to the combination effect on MVD. IL-6 production was greatly reduced by erlotinib in the erlotinib-sensitive phase, whereas a slight but statistically significant increase in IL-6 production was observed in the erlotinib-refractory phase. Since IL-6 is reported to have pro-angiogenic activity either directly by acting on vascular endothelial cells or indirectly via STAT3induced VEGF production $(34,35)$, the contribution of IL-6 on angiogenesis as well as tumor cell STAT3 activation in our model are to be further investigated.

Our findings suggested that VEGF was an inducer of growth signals through VEGFR- and/or VEGF-mediated production of tumor/host growth factors in our model. Several studies have reported that VEGFRs are expressed in many human tumor types and that VEGF is an autocrine growth factor for tumor cell lines that express VEGFRs (36-38). On the other hand, tumor cells exist within a complex microenvironment comprising numerous cells including, for example, vascular endothelial cells of the blood and lymphatic circulatory system, stromal fibroblasts, tumor associated macrophages (TAMs), and myeloid derived suppressor cells (MDSCs). Furthermore, paracrine signaling interactions between tumor cells and stromal cells are a key component in the proliferation of tumors in several organs $(39,40)$. Cumulative evidence shows that VEGF can induce tumor growth, not only by promoting angiogenesis but also by creation of other favorable tumor microenvironments (41). TAMs express VEGFR2, and selective inhibition of VEGFR2 reduces recruitment of macrophages into orthotopic pancreatic tumors (42), and treatment with sunitinib, a receptor tyrosine kinase inhibitor, decreased the number of MDSCs in advanced tumor-bearing animals (43). A

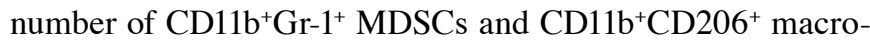
phages infiltrated into tumors in our model (data not shown). Therefore, VEGF secreted by the tumor may activate tumor growth directly or indirectly through host cells including MDSC and TAM.

In conclusion, continuous treatment with erlotinib plus bevacizumab shows promising efficacy in the B901L xenograft model of $E G F R$ Mut $^{+}$NSCLC. Furthermore, re-induction of VEGF and subsequent VEGF-dependent tumor growth is suggested as one of the major mechanisms of acquired resistance to erlotinib. Therefore, remarkably prolonged antitumor activity was achieved by inhibition of VEGF by bevacizumab in combination with erlotinib. In this study, we established a model that became refractory to erlotinib after long-term administration of erlotinib and in which prolonged antitumor activity was shown by treatment with erlotinib plus bevacizumab, which is in line with the results of the Phase II trial (JO25567) to evaluate the efficacy of erlotinib plus bevacizumab in EGFR $\mathrm{Mut}^{+}$NSCLC patients. A Phase III trial (NEJ026) is currently underway. Further studies are required to better elucidate the mechanisms of action of erlotinib plus bevacizumab in NSCLC harboring EGFR mutations. 


\section{Acknowledgements}

The authors thank Masako Miyazaki, Kumiko Kondoh, and Hiromi Sawamura at Chugai for their technical assistance with drug administration, and also thank Dr Yoriko YamashitaKashima, Dr Kazushige Mori, and Dr Kaori Fujimoto-Ouchi for their helpful support and advice in this study.

\section{References}

1. Shepherd FA, Rodrigues Pereira J, Ciuleanu T, Tan EH, Hirsh V, Thongprasert S, Campos D, Maoleekoonpiroj S, Smylie M, Martins R, et al; National Cancer Institute of Canada Clinical Trials Group: Erlotinib in previously treated non-small-cell lung cancer. N Engl J Med 353: 123-132, 2005.

2. Zhou C, Wu YL, Chen G, Feng J, Liu XQ, Wang C, Zhang S, Wang J, Zhou S, Ren S, et al: Erlotinib versus chemotherapy as first-line treatment for patients with advanced EGFR mutationpositive non-small-cell lung cancer (OPTIMAL, CTONG-0802) A multicentre, open-label, randomised, phase 3 study. Lancet Oncol 12: 735-742, 2011.

3. Rosell R, Carcereny E, Gervais R, Vergnenegre A, Massuti B, Felip E, Palmero R, Garcia-Gomez R, Pallares C, Sanchez JM, et al; Spanish Lung Cancer Group in collaboration with Groupe Français de Pneumo-Cancérologie and Associazione Italiana Oncologia Toracica: Erlotinib versus standard chemotherapy as first-line treatment for European patients with advanced EGFR mutation-positive non-small-cell lung cancer (EURTAC): A multicentre, open-label, randomised phase 3 trial. Lancet Oncol 13: 239-246, 2012

4. Pao W, Miller VA, Politi KA, Riely GJ, Somwar R, Zakowski MF, Kris MG and Varmus H: Acquired resistance of lung adenocarcinomas to gefitinib or erlotinib is associated with a second mutation in the EGFR kinase domain. PLoS Med 2: e73, 2005.

5. Yu HA, Arcila ME, Rekhtman N, Sima CS, Zakowski MF, Pao W, Kris MG, Miller VA, Ladanyi M and Riely GJ: Analysis of tumor specimens at the time of acquired resistance to EGFR-TKI therapy in 155 patients with EGFR-mutant lung cancers. Clin Cancer Res 19: 2240-2247, 2013.

6. Yuan F, Chen Y, Dellian M, Safabakhsh N, Ferrara N and Jain RK: Time-dependent vascular regression and permeability changes in established human tumor xenografts induced by an anti-vascular endothelial growth factor/vascular permeability factor antibody. Proc Natl Acad Sci USA 93: 14765-14770, 1996.

7. O'Connor JP, Carano RA, Clamp AR, Ross J, Ho CC, Jackson A, Parker GJ, Rose CJ, Peale FV, Friesenhahn M, et al: Quantifying antivascular effects of monoclonal antibodies to vascular endothelial growth factor: Insights from imaging. Clin Cancer Res 15: 6674-6682, 2009.

8. Gerber HP and Ferrara N: Pharmacology and pharmacodynamics of bevacizumab as monotherapy or in combination with cytotoxic therapy in preclinical studies. Cancer Res 65: 671-680, 2005.

9. Yanagisawa M, Yorozu K, Kurasawa M, Nakano K, Furugaki K, Yamashita Y, Mori K and Fujimoto-Ouchi K: Bevacizumab improves the delivery and efficacy of paclitaxel. Anticancer Drugs 21: 687-694, 2010.

10. Turley RS, Fontanella AN, Padussis JC, Toshimitsu H, Tokuhisa Y, Cho EH, Hanna G, Beasley GM, Augustine CK, Dewhirst MW, et al: Bevacizumab-induced alterations in vascular permeability and drug delivery: A novel approach to augment regional chemotherapy for in-transit melanoma. Clin Cancer Res 18: 3328-3339, 2012.

11. Sandler A, Gray R, Perry MC, Brahmer J, Schiller JH, Dowlati A, Lilenbaum R and Johnson DH: Paclitaxel-carboplatin alone or with bevacizumab for non-small-cell lung cancer. N Engl J Med 355: 2542-2550, 2006

12. Herbst RS, Johnson DH, Mininberg E, Carbone DP, Henderson T, Kim ES, Blumenschein G Jr, Lee JJ, Liu DD, Truong MT, et al: Phase I/II trial evaluating the anti-vascular endothelial growth factor monoclonal antibody bevacizumab in combination with the HER-1/epidermal growth factor receptor tyrosine kinase inhibitor erlotinib for patients with recurrent non-small-cell lung cancer. J Clin Oncol 23: 2544-2555, 2005.
13. Camp ER, Summy J, Bauer TW, Liu W, Gallick GE and Ellis LM: Molecular mechanisms of resistance to therapies targeting the epidermal growth factor receptor. Clin Cancer Res 11: 397-405, 2005.

14. Seto T, Kato T, Nishio M, Goto K, Atagi S, Hosomi Y, Yamamoto N, Hida T, Maemondo M, Nakagawa K, et al: Erlotinib alone or with bevacizumab as first-line therapy in patients with advanced non-squamous non-small-cell lung cancer harbouring EGFR mutations (JO25567): An open-label, randomised, multicentre, phase 2 study. Lancet Oncol 15: 1236-1244, 2014.

15. Li H, Takayama K, Wang S, Shiraishi Y, Gotanda K, Harada T, Furuyama K, Iwama E, Ieiri I, Okamoto I, et al: Addition of bevacizumab enhances antitumor activity of erlotinib against non-small cell lung cancer xenografts depending on VEGF expression. Cancer Chemother Pharmacol 74: 1297-1305, 2014.

16. Pore N, Jiang Z, Gupta A, Cerniglia G, Kao GD and Maity A: EGFR tyrosine kinase inhibitors decrease VEGF expression by both hypoxia-inducible factor (HIF)-1-independent and HIF-1dependent mechanisms. Cancer Res 66: 3197-3204, 2006.

17. Lee JG and Wu R: Erlotinib-cisplatin combination inhibits growth and angiogenesis through c-MYC and HIF-1 $\alpha$ in EGFRmutated lung cancer in vitro and in vivo. Neoplasia 17: 190-200, 2015.

18. Tabernero J: The role of VEGF and EGFR inhibition: Implications for combining anti-VEGF and anti-EGFR agents. Mol Cancer Res 5: 203-220, 2007.

19. Chatterjee S, Wieczorek C, Schöttle J, Siobal M, Hinze Y, Franz T, Florin A, Adamczak J, Heukamp LC, Neumaier B, et al: Transient antiangiogenic treatment improves delivery of cytotoxic compounds and therapeutic outcome in lung cancer. Cancer Res 74: 2816-2824, 2014.

20. Furugaki K, Yasuno H, Iwai T, Moriya Y, Harada N and Fujimoto-Ouchi K: Melting curve analysis for mutations of EGFR and KRAS. Anticancer Res 34: 613-621, 2014.

21. Furugaki K, Fukumura J, Iwai T, Yorozu K, Kurasawa M, Yanagisawa M, Moriya Y, Yamamoto K, Suda K, Mizuuchi H, et al: Impact of bevacizumab in combination with erlotinib on EGFR-mutated non-small cell lung cancer xenograft models with T790M mutation or MET amplification. Int J Cancer 138: 1024-1032, 2016

22. Nakade J, Takeuchi S, Nakagawa T, Ishikawa D, Sano T, Nanjo S, Yamada T, Ebi H, Zhao L, Yasumoto K, et al: Triple inhibition of EGFR, Met, and VEGF suppresses regrowth of HGF-triggered, erlotinib-resistant lung cancer harboring an EGFR mutation. J Thorac Oncol 9: 775-783, 2014.

23. Larsen AK, Ouaret D, El Ouadrani K and Petitprez A: Targeting EGFR and $\operatorname{VEGF(R)}$ pathway cross-talk in tumor survival and angiogenesis. Pharmacol Ther 131: 80-90, 2011.

24. Petit AM, Rak J, Hung MC, Rockwell P, Goldstein N, Fendly B and Kerbel RS: Neutralizing antibodies against epidermal growth factor and ErbB-2/neu receptor tyrosine kinases down-regulate vascular endothelial growth factor production by tumor cells in vitro and in vivo: Angiogenic implications for signal transduction therapy of solid tumors. Am J Pathol 151: 1523-1530, 1997.

25. Yen L, You XL, Al Moustafa AE, Batist G, Hynes NE, Mader S, Meloche S and Alaoui-Jamali MA: Heregulin selectively upregulates vascular endothelial growth factor secretion in cancer cells and stimulates angiogenesis. Oncogene 19: 3460-3469, 2000.

26. Viloria-Petit A, Crombet T, Jothy S, Hicklin D, Bohlen P, Schlaeppi JM, Rak J and Kerbel RS: Acquired resistance to the antitumor effect of epidermal growth factor receptor-blocking antibodies in vivo: A role for altered tumor angiogenesis. Cancer Res 61: 5090-5101, 2001.

27. Naumov GN, Nilsson MB, Cascone T, Briggs A, Straume O, Akslen LA, Lifshits E, Byers LA, Xu L, Wu HK, et al: Combined vascular endothelial growth factor receptor and epidermal growth factor receptor (EGFR) blockade inhibits tumor growth in xenograft models of EGFR inhibitor resistance. Clin Cancer Res 15: 3484-3494, 2009.

28. Schicher N, Paulitschke V, Swoboda A, Kunstfeld R, Loewe R, Pilarski P, Pehamberger $\mathrm{H}$ and Hoeller C: Erlotinib and bevacizumab have synergistic activity against melanoma. Clin Cancer Res 15: 3495-3502, 2009.

29. Herbst RS and Sandler A: Bevacizumab and erlotinib: A promising new approach to the treatment of advanced NSCLC. Oncologist 13: 1166-1176, 2008.

30. Zhu AX, Duda DG, Sahani DV and Jain RK: HCC and angiogenesis: Possible targets and future directions. Nat Rev Clin Oncol 8: 292-301, 2011. 
31. Shojaei F, Wu X, Qu X, Kowanetz M, Yu L, Tan M, Meng YG and Ferrara N: G-CSF-initiated myeloid cell mobilization and angiogenesis mediate tumor refractoriness to anti-VEGF therapy in mouse models. Proc Natl Acad Sci USA 106: 6742-6747, 2009.

32. Vandercappellen J, Van Damme J and Struyf S: The role of CXC chemokines and their receptors in cancer. Cancer Lett 267: 226-244, 2008.

33. Strieter RM, Burdick MD, Gomperts BN, Belperio JA and Keane MP: CXC chemokines in angiogenesis. Cytokine Growth Factor Rev 16: 593-609, 2005.

34. Wei LH, Kuo ML, Chen CA, Chou CH, Lai KB, Lee CN and Hsieh CY: Interleukin-6 promotes cervical tumor growth by VEGF-dependent angiogenesis via a STAT3 pathway. Oncogene 22: 1517-1527, 2003.

35. Nilsson MB, Langley RR and Fidler IJ: Interleukin-6, secreted by human ovarian carcinoma cells, is a potent proangiogenic cytokine. Cancer Res 65: 10794-10800, 2005.

36. Goel HL and Mercurio AM: VEGF targets the tumour cell. Nat Rev Cancer 13: 871-882, 2013.

37. Barr MP, Gray SG, Gately K, Hams E, Fallon PG, Davies AM, Richard DJ, Pidgeon GP and O'Byrne KJ: Vascular endothelial growth factor is an autocrine growth factor, signaling through neuropilin-1 in non-small cell lung cancer. Mol Cancer 14: 45 , 2015.
38. Masood R, Cai J, Zheng T, Smith DL, Hinton DR and Gill PS: Vascular endothelial growth factor (VEGF) is an autocrine growth factor for VEGF receptor-positive human tumors. Blood 98: 1904-1913, 2001.

39. Joyce JA and Pollard JW: Microenvironmental regulation of metastasis. Nat Rev Cancer 9: 239-252, 2009.

40. Murdoch C, Muthana M, Coffelt SB and Lewis CE: The role of myeloid cells in the promotion of tumour angiogenesis. Nat Rev Cancer 8: 618-631, 2008.

41. Voron T, Marcheteau E, Pernot S, Colussi O, Tartour E, Taieb J and Terme M: Control of the immune response by pro-angiogenic factors. Front Oncol 4: 70, 2014.

42. Dineen SP, Lynn KD, Holloway SE, Miller AF, Sullivan JP, Shames DS, Beck AW, Barnett CC, Fleming JB and Brekken RA: Vascular endothelial growth factor receptor 2 mediates macrophage infiltration into orthotopic pancreatic tumors in mice. Cancer Res 68: 4340-4346, 2008.

43. Ozao-Choy J, Ma G, Kao J, Wang GX, Meseck M, Sung M, Schwartz M, Divino CM, Pan PY and Chen SH: The novel role of tyrosine kinase inhibitor in the reversal of immune suppression and modulation of tumor microenvironment for immune-based cancer therapies. Cancer Res 69: 2514-2522, 2009. 